\title{
Alterations of cellular aging markers in obsessive- compulsive disorder: mitochondrial DNA copy number and telomere length
}

\author{
Jee In Kang, MD, PhD; Chun Il Park, MD; Jue Lin, PhD; Shin Tae Kim, MD; \\ Hae Won Kim, MD, PhD; Se Joo Kim, MD, PhD
}

\begin{abstract}
Background: The present study examined whether mitochondrial DNA copy number (mtDNAcn) and telomere length — key markers of cellular aging - were altered in male and female participants with obsessive-compulsive disorder (OCD) compared to healthy controls. We also tested for associations between these alterations and OCD-related clinical features and inflammatory index. Methods: A total of 235 patients with OCD $(38.7 \%$ female) and 234 healthy controls $(41.5 \%$ female) were included. We quantified whole-blood mtDNAcn and leukocyte telomere length using quantitative polymerase chain reaction. We also calculated the neutrophil-to-lymphocyte ratio from complete blood cell counts. Results: Multivariate analysis of covariance showed that OCD status had a significant overall effect on cellular aging markers in men (Wilks $\lambda=0.889, F_{2,275}=17.13, p<0.001$ ) and women (Wilks $\lambda=0.742, F_{2,182}=31.61, p<0.001$ ) after controlling for age, body mass index and childhood trauma. In post-hoc comparisons, men with OCD had lower mtDNAcn than controls $(p<0.001)$, but we found no between-group difference for telomere length $(p=0.55)$. Women with OCD had a significantly lower mtDNAcn $(p<$ $0.001)$ and shortened telomere length $(p=0.023)$ compared to controls. Moreover, the lower mtDNAcn shown in the OCD group was significantly correlated with an increase in systemic inflammation for both sexes, as measured by neutrophil-to-lymphocyte ratio. Limitations: The present cross-sectional design did not allow us to infer a causal relationship between OCD disease status and cellular aging markers. Conclusion: The present study is, to our knowledge, the first to demonstrate alterations in mtDNAcn and telomere shortening in OCD. These results suggest that aging-associated molecular mechanisms may be important in the pathophysiology of OCD.
\end{abstract}

\section{Introduction}

Obsessive-compulsive disorder (OCD) is a chronic neuropsychiatric disorder characterized by repetitive, intrusive and time-consuming thoughts (obsessions) and irresistible irrational behaviours or rituals (compulsions), which can cause severe distress to the patient. ${ }^{1}$ People with OCD often identify traumatic events and psychosocial stress as factors that develop and exacerbate their symptoms. ${ }^{2}$ In addition, cross-sectional case-control studies have shown oxidative imbalance $^{3,4}$ and oxidative DNA damage ${ }^{5}$ in patients with OCD, suggesting that oxidative stress may play a crucial role in the pathophysiology of OCD. Although the effects of stress on the pathophysiology of OCD remain unclear, chronic stress may influence the underlying biochemical processes and neurobiological changes that occur in OCD.

Recently, the association of cellular aging with chronic stress and neuropsychiatric conditions has become a growing field of research. People with serious mental illnesses are prone to various physical health problems. ${ }^{6}$ Physical morbidities such as cardiovascular and metabolic diseases were more prevalent in patients with psychiatric disorders than in the general population. ${ }^{7}$ Recent research has shown that OCD is associated with an increased risk of allergies and endocrine diseases. ${ }^{8,9}$ Such comorbidity for physical diseases and psychiatric disorders may be associated with accelerated cellular aging. ${ }^{10}$ However, few studies have investigated the role of cellular aging in OCD.

Accumulating evidence has shown that the shortening of telomere length in response to cumulative oxidative stress ${ }^{11,12}$ is implicated in accelerated cellular aging and increased physical morbidity in neuropsychiatric conditions. ${ }^{13,14}$ As well, mitochondrial function has attracted much attention as a biomarker of stress adaptation and biological aging in chronic stress and psychiatric disorders. ${ }^{15}$ Mitochondria highly dynamic organelles located in the cytosol of eukaryotic cells - play a key role in cellular energy production and homeostasis by producing adenosine triphosphate through

Correspondence to: S. J. Kim, Department of Psychiatry, Yonsei University College of Medicine, Yonsei-ro 50-1, Seodaemun-gu, Seoul, South Korea, 03722; kimsejoo@yuhs.ac

Submitted Dec. 23, 2020; Revised Feb. 26, 2021; Accepted Mar. 17, 2021

DOI: $10.1503 / j p n .200238$ 
oxidative phosphorylation, and their fission and fusion processes help to facilitate adaptation to the changing environment in response to metabolic demand or stress. ${ }^{16}$ Altered mitochondrial DNA copy number (mtDNAcn), an index of mitochondrial dysfunction, has been associated with several psychiatric disorders, including posttraumatic stress disorder ${ }^{17}$ and bipolar disorder, ${ }^{18}$ although there have also been mixed findings of higher, reduced or unchanged mtDNAcn in psychiatric disorders. To date, no study has investigated whether telomere shortening or altered mtDNAcn occur in OCD.

In the present study, we examined whether leukocyte telomere length and mtDNAcn were altered in male and female participants with OCD compared to healthy controls. We also tested whether these markers were associated with clinical and inflammatory factors of OCD. Given the role of stress and the possible involvement of oxidative stress in the pathogenesis of OCD, we hypothesized that participants with OCD would exhibit telomere shortening and altered mtDNAcn compared to healthy controls, and that the alterations would be associated with clinical features and an inflammatory index of OCD.

\section{Methods}

\section{Participants}

A total of 243 patients with OCD (94 women) and 241 healthy controls (99 women) were included in the present study. The patients with OCD were recruited at an OCD-specialized outpatient clinic at Severance Hospital, Yonsei University Health System (Seoul, Republic of Korea). Healthy controls were recruited through advertisements. All participants were assessed by trained psychiatrists using the Structured Clinical Interview for the DSM-IV ${ }^{19}$ to evaluate them for the presence of current and previous psychiatric disorders. To be included in the OCD group, people had to be aged 19 to 50 years and have a primary diagnosis of OCD. A psychiatrist (S.J. Kim) confirmed the diagnosis based on the DSM-IV. ${ }^{19}$ Exclusion criteria were psychosis, bipolar disorder, substance dependence, mental retardation as defined by the DSM-IV, head trauma, major somatic and neurologic disorders, pregnancy and breastfeeding. The ethnicity of all participants was selfreported and only those who were ethnically Korean were enrolled. All participants provided written informed consent before participation, and this study was approved by the institutional review board of Severance Hospital, Yonsei University Health System.

\section{Measurement of telomere length and mtDNAcn in blood}

Genomic DNA was isolated from peripheral blood samples using a nucleic acid isolation device, the QuickGene-mini80 (Fujifilm). Telomere length and mtDNAcn were then measured in the Blackburn Laboratory at the University of California, San Francisco. Telomere length (relative telomere to single copy gene ratio) was measured using quantitative polymerase chain reaction ( $\mathrm{qPCR}$ ) based on a method originally published by Cawthon..$^{20}$ DNA samples were assayed twice in triplicate wells. If the difference between the 2 runs was greater than $7 \%$, a third run was conducted and the average of the closest values was reported. The inter-assay coefficient of variation for telomere length was $2.1 \%$. A detailed description of the assay procedures has been described previously. ${ }^{21}$

For mtDNAcn, the relative copy number of mtDNA per diploid nuclear genome was determined using a TaqMan multiplex assay. This involved the detection of a $69 \mathrm{bp}$ fragment of the ND1 gene in mtDNA (nucleotides 3485-3553) and an 87 bp fragment of RNase P (TaqMan Copy Number Reference Assay, human, RNase P; cat. no. 4403328; Life Technologies) in the nuclear genomic DNA, based on a previously published method. ${ }^{22}$ DNA samples were assayed in triplicate wells in a single run. A subset of samples was randomly selected for a second run to obtain the inter-assay coefficient of variation, which was $4.1 \%$ for mtDNA. Details of the assay procedures have been previously described. ${ }^{17}$ All qPCR tests were performed using the LightCycler 480 Instrument II (Roche Diagnostics).

\section{Measures of clinical characteristics}

The clinical symptoms of OCD were evaluated by a trained psychologist using the 10-item Yale-Brown Obsessive Compulsive $\mathrm{Scale}^{23}$ for obsessive-compulsive symptom severity and the Montgomery-Åsberg Depression Rating Scale ${ }^{24}$ for depressive symptoms.

Age at onset of OCD was considered to be the age at which obsessive or compulsive symptoms first occurred as remembered by patients or family members and was dichotomized as early-onset ( $\leq 17$ years) and late-onset ( $>17$ years). ${ }^{25}$

Childhood traumatic experiences were assessed using the Early Trauma Inventory-Self-Report Short Form, ${ }^{26}$ which consists of 27 items in 4 different domains of childhood traumatic experiences: general trauma and physical, sexual and emotional abuse. The scores for all 4 domains are summed.

\section{Measure of neutrophil-to-lymphocyte ratio as an inflammatory marker}

We analyzed the complete blood count with differential white blood cell count in a subset of the participants with OCD $(n=202)$ using the XN-20 (Sysmex). We calculated the neutrophil-to-lymphocyte ratio (NLR), an easily measured marker associated with systemic inflammation, ${ }^{27}$ from the complete blood count. Participants with a white blood cell count greater than 10000 cells $/ \mu \mathrm{L}(n=18)$ or those with extreme NLR outliers $(n=2)$ were excluded from our analyses.

\section{Statistical analyses}

We compared demographic and clinical characteristics between groups using independent-samples $t$ tests for continuous variables and Pearson $\chi^{2}$ tests for categorical variables. We calculated Pearson correlation $(r)$ and partial correlation (pr) coefficients to examine relationships between the cellular markers and other variables. We performed multivariate 
analysis of covariance (MANCOVA) and post-hoc comparisons using the 2 cellular markers as dependent variables and OCD status as an independent variable. Because lifespan and aging-related markers such as telomere length or attrition rate are affected by sex differences, ${ }^{28}$ we performed MANCOVAs in sex-stratified analyses to control for the possible effect of sex difference and reduce potential bias related to unmeasured interaction effects between sex and clinical features. In the partial correlation analysis and MANCOVAs, we controlled for potential confounders (age, body mass index and childhood trauma) that have been associated with cellular aging and oxidative stress. ${ }^{29,30}$ As well, to examine the relationships between the 2 markers (telomere length and mtDNAcn) and clinical characteristics of OCD, we conducted hierarchical multiple linear regression analyses with 2 steps in each sex. In these analyses, we entered age, body mass index and childhood trauma as a block in the first step, and then 4 clinical characteristics (age at onset, duration of illness, Yale-Brown Obsessive Compulsive Scale score and Montgomery-Åsberg Depression Rating Scale score) as a second block in the next step. We analyzed all data using the Statistical Package for the Social Sciences, version 25.0 (SPSS Inc.). All tests were 2-tailed, and $p<0.025$ was considered statistically significant based on Bonferroni correction for 2 dependent variables. Data values are presented as mean \pm standard deviation.

\section{Results}

\section{Sample characteristics}

Of those who participated in the interview (243 patients with OCD and 241 controls), data from 8 patients and 7 controls were excluded from the final analysis: 7 controls had comorbid psychiatric disorders, 5 patients with OCD had incomplete self-reported data, 2 samples contained insufficient DNA, and 1 patient had an outlier telomere length value. The final analyses included 235 patients with OCD (91 women) and 234 healthy controls (97 women).
The mean ages for the OCD and control groups were 27.70 \pm 7.55 years and $27.23 \pm 7.72$ years, respectively. We found no significant differences between the groups with respect to age, sex, education status or body mass index. Based on Early Trauma Inventory total scores, those in the OCD group had experienced more childhood trauma than those in the control group ( $p<0.001 ;$ Table 1$)$.

\section{Demographic and psychosocial factors associated with telomere length and mtDNAcn}

In correlation analyses for the entire study population, age was inversely correlated with telomere length $(r=-0.303, p<$ $0.001)$, but was not significantly correlated with mtDNAcn $(r=-0.006, p=0.89)$. Childhood trauma based on Early Trauma Inventory total score had a weak negative correlation with mtDNAcn $(r=-0.094, p=0.04)$ but not telomere length $(r=-0.031, p=0.50)$. As well, mtDNAcn was positively associated with telomere length $(r=0.179, p<0.001)$. When controlling for age, body mass index and childhood trauma, the correlation between mtDNAcn and telomere length remained significant for the entire study population $(p r=0.182, p<$ 0.001 ) and for the control and OCD groups (healthy controls $p r=0.153, p=0.020$; patients with OCD $p r=0.206, p=0.002$ ).

Among healthy controls, women exhibited higher telomere length (women $1.099 \pm 0.225 \mathrm{v}$. men $1.043 \pm 0.225, p=0.04$ ) and higher mtDNAcn (women $551.7 \pm 103.1 \mathrm{v}$. men $519.5 \pm$ $119.5, p=0.03$ ) than men. Among patients with OCD, neither telomere length (women $1.030 \pm 0.184 \mathrm{v}$. men $1.050 \pm 0.225$, $p=0.43$ ) nor mtDNAcn (women $423.6 \pm 124.5 \mathrm{v}$. men: $434.3 \pm$ $116.2, p=0.51$ ) was significantly different between women and men.

\section{MANCOVA}

MANCOVA using age, body mass index and childhood trauma as covariates showed that OCD status had a significant overall effect on mtDNAcn and relative telomere

Table 1: Demographic and clinical characteristics of patients with OCD and healthy controls

\begin{tabular}{|c|c|c|c|c|}
\hline Characteristic & $\begin{array}{c}\text { OCD* }^{*} \\
(n=235)\end{array}$ & $\begin{array}{l}\text { Healthy controls } \\
\qquad(n=234)\end{array}$ & $t$ or $\chi^{2}$ & $p$ value \\
\hline Age, yr & $27.70 \pm 7.55$ & $27.23 \pm 7.72$ & 0.668 & 0.50 \\
\hline Education, high school/college or higher & $126 / 109$ & $123 / 111$ & 0.052 & 0.82 \\
\hline Male/female & $144 / 91$ & $137 / 97$ & 0.364 & 0.55 \\
\hline Body mass index, $\mathrm{kg} / \mathrm{m}^{2}$ & $23.07 \pm 2.79$ & $22.87 \pm 3.41$ & 0.716 & 0.47 \\
\hline Early Trauma Inventory-Self-Report Short Form score & $6.70 \pm 4.77$ & $4.19 \pm 3.86$ & 6.268 & $<0.001$ \\
\hline Yale-Brown Obsessive-Compulsive Scale score & $25.79 \pm 6.83$ & $0.47 \pm 1.87$ & 54.63 & $<0.001$ \\
\hline Montgomery-Åsberg Depression Rating Scale score & $20.54 \pm 9.94$ & $3.29 \pm 3.95$ & 24.72 & $<0.001$ \\
\hline Age at onset, yr & $18.06 \pm 7.30$ & - & & \\
\hline Early onset/late onset & $119 / 116$ & - & & \\
\hline Duration of illness, yr & $9.69 \pm 6.74$ & - & & \\
\hline \multicolumn{5}{|l|}{ Cellular aging markers } \\
\hline Relative telomere length & $1.042 \pm 0.185$ & $1.066 \pm 0.198$ & -1.357 & 0.18 \\
\hline Mitochondrial DNA copy number & $430.12 \pm 119.34$ & $532.85 \pm 113.88$ & -9.537 & $<0.001$ \\
\hline
\end{tabular}


length in both men (Wilks $\lambda=0.889, F_{2,275}=17.13, p<0.001$ ) and women (Wilks $\lambda=0.742, F_{2,182}=31.61, p<0.001$; Table 2). In post-hoc comparisons, male participants with OCD had a significantly reduced mtDNAcn compared to healthy controls $(p<0.001)$, but we found no significant difference in relative telomere length between the 2 groups $(p=0.55)$. In contrast, female participants with OCD had a significantly reduced mtDNAcn $(p<0.001)$ and a shortened telomere length $(p=0.023)$ compared to healthy controls (Table 2 and Figure 1).

\section{Medication in patients with $O C D$}

Of the 144 men with OCD, 45 (31.3\%) were drug-naive or had been drug-free for more than 8 weeks; the other 99 $(68.7 \%)$ had been prescribed serotonin reuptake inhibitors. Of the 91 women with OCD, 32 (35.2\%) were drug-naive or drug-free for more than 8 weeks, and 59 (64.8\%) were taking serotonin reuptake inhibitors. We found no significant difference between unmedicated and medicated patients with respect to telomere length (women $1.064 \pm 0.204$ v. $1.012 \pm 0.172, p=0.20$; men $1.072 \pm 0.193$ v. $1.039 \pm 0.181$, $p=0.33$ ) or mtDNAcn (women $446.3 \pm 120.6$ v. $411.3 \pm$ $125.9, p=0.20$; men $430.5 \pm 111.0$ v. $436.0 \pm 119.0, p=0.33)$ in either sex.
Association between mtDNAcn and telomere length and clinical characteristics of OCD

Hierarchical multiple linear regression analyses showed no significant associations between mtDNAcn or telomere length and symptom severity of OCD, depressive symptoms, age at onset or duration of illness in participants with OCD of either sex.

Relationship of an inflammatory factor (NLR) with mtDNAcn and telomere length in men and women with OCD

In partial correlation analyses with covariates of age, body mass index and childhood trauma, we found a significant association between NLR and mtDNAcn in both men and women with OCD (men $[n=115] p r=-0.330, p<0.001$; women $[n=67] \mathrm{pr}=-0.519, p<0.001)$. Figure 2 shows a scatterplot of raw scores for the 2 variables, which were inversely correlated in each sex. We found no significant association between NLR and telomere length for either sex.

\section{Discussion}

In the present study, we investigated whether leukocyte telomere length and whole-blood mtDNAcn were

Table 2: MANCOVA of leukocyte telomere length and mitochondrial DNA copy number data from male and female OCD and control groups

\begin{tabular}{|c|c|c|c|c|c|c|}
\hline \multirow[b]{2}{*}{ Result } & \multicolumn{2}{|c|}{$\operatorname{Men}(n=281)$} & \multirow[b]{2}{*}{$p$ value } & \multicolumn{2}{|c|}{ Women $(n=188)$} & \multirow[b]{2}{*}{$p$ value } \\
\hline & $\begin{array}{c}\text { OCD } \\
(n=144)\end{array}$ & $\begin{array}{l}\text { Healthy controls } \\
\qquad(n=137)\end{array}$ & & $\begin{array}{c}\text { OCD } \\
(n=91)\end{array}$ & $\begin{array}{l}\text { Healthy controls } \\
\qquad(n=97)\end{array}$ & \\
\hline MANCOVA* $^{*}$ & \multicolumn{2}{|c|}{ Wilks $\lambda=0.889 ; F_{2,275}=17.13$} & $<0.001$ & \multicolumn{2}{|c|}{ Wilks $\lambda=0.742 ; F_{2,182}=31.61$} & $<0.001$ \\
\hline Leukocyte telomere length & $1.050 \pm 0.185$ & $1.043 \pm 0.173$ & 0.55 & $1.030 \pm 0.184$ & $1.099 \pm 0.225$ & 0.023 \\
\hline Mitochondrial DNA copy number & $434.25 \pm 116.19$ & $519.49 \pm 119.48$ & $<0.001$ & $423.58 \pm 124.54$ & $551.73 \pm 103.14$ & $<0.001$ \\
\hline
\end{tabular}

A



B

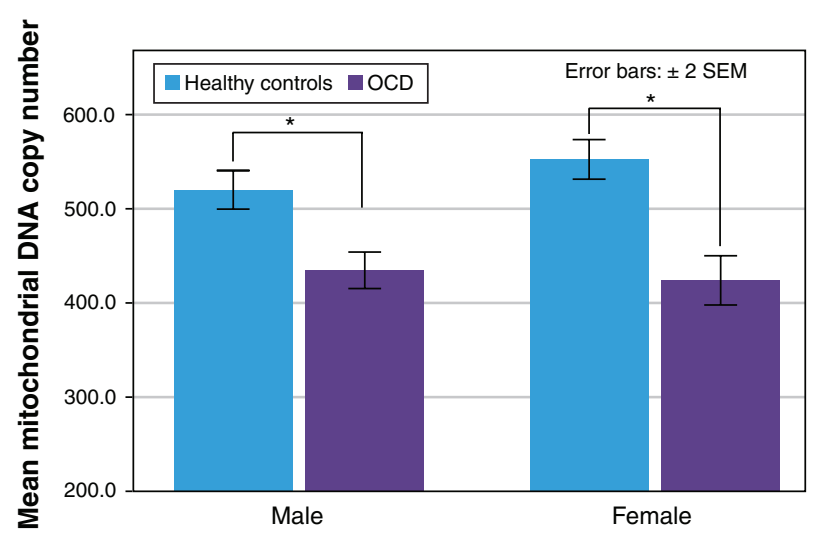

Figure 1: Relative leukocyte telomere length and whole blood mitochondrial DNA copy number for male and female patients with OCD and healthy controls. ${ }^{*} p<0.05$. Error bars represent \pm 2 standard errors of the mean. $\mathrm{OCD}=$ obsessive-compulsive disorder; $\mathrm{SEM}=\mathrm{standard}$ error of the mean. 


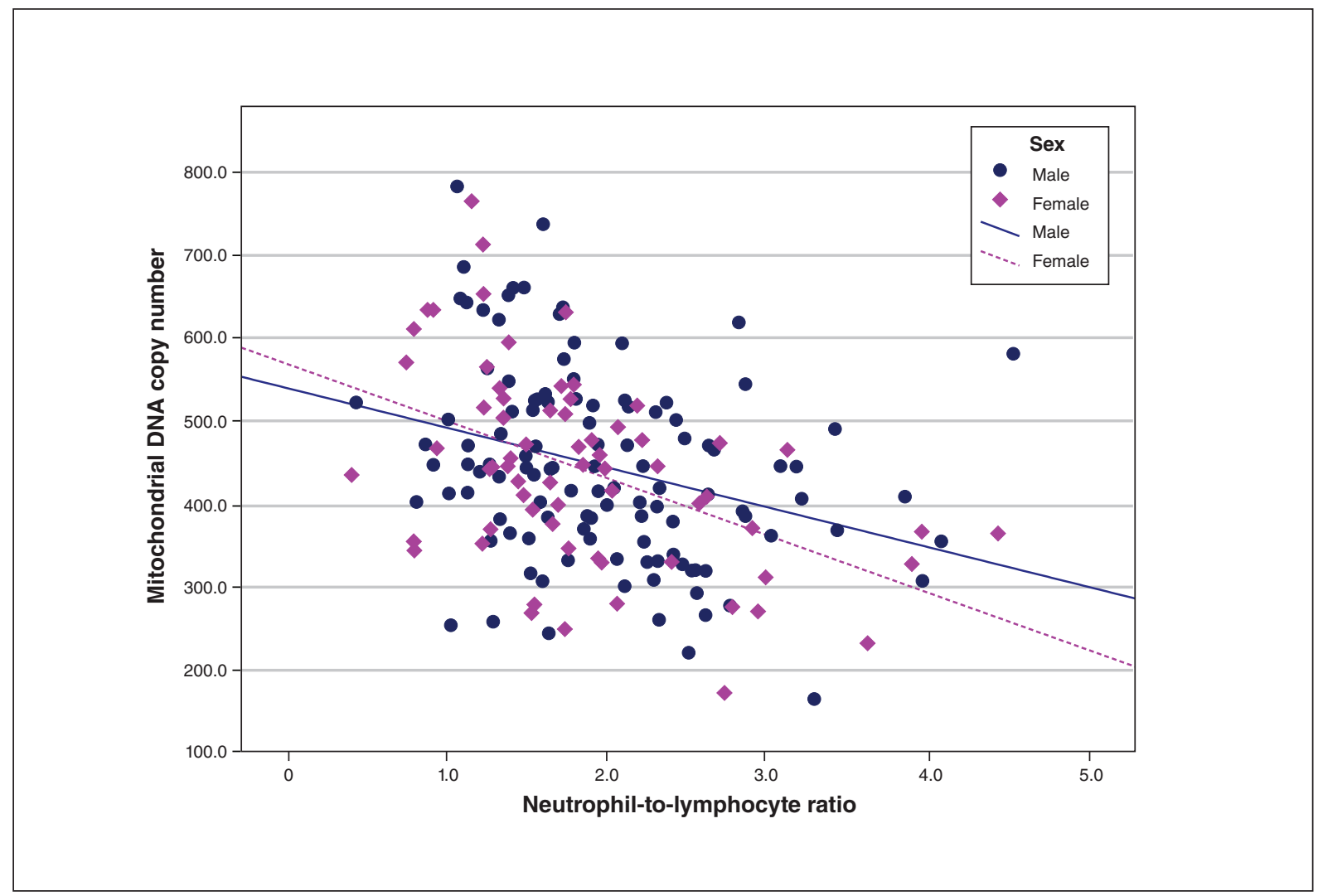

Figure 2: Association between neutrophil-to-lymphocyte ratio and mitochondrial DNA copy number. Using original raw data without adjusting for covariates, the scatterplot shows an inverse relationship for both sexes.

associated with OCD in a relatively homogeneous Korean population. To the best of our knowledge, this was the first study to investigate the role of telomere length and mtDNAcn in the pathophysiology of OCD. We demonstrated significantly reduced telomere length and mtDNAcn in female participants with OCD compared to healthy controls. Male participants with OCD also had a significantly reduced mtDNAcn compared to healthy controls, but we found no significant difference in telomere length between the 2 groups. These results suggest the possible involvement of mitochondrial dysfunction and telomere erosion in the pathophysiology of OCD.

We demonstrated a reduction in mtDNAcn in both male and female participants with OCD. The mtDNAcn in the present OCD sample showed no significant associations with clinical variables such as duration of illness or YaleBrown Obsessive Compulsive Scale, possibly because of a lack of statistical power to detect an association; another possible explanation is that mtDNAcn may be a predisposing factor for OCD rather than a state factor of clinical features. Although little is known about whether OCD is associated with altered $\mathrm{mtDNAcn}$, there has been indirect evidence of mitochondrial involvement in OCD. Occurrence of OCD has been reported in cases with mitochondrial disease. ${ }^{31}$ In addition, genetic variants associated with mitochondrial disease have been identified in patients with OCD. ${ }^{32} \mathrm{Al}-$ though the underlying mechanisms linking mitochondrial dysfunction to OCD are unclear, a growing body of evidence suggests that the role of mitochondria in cellular stress responses and regulation of energy metabolism and homeostasis may be important in pathophysiological changes of the brain to stress. ${ }^{33}$ Notably, mitochondria are principal hubs in the regulation of immune and inflammatory responses. ${ }^{34}$ A study in Taiwanese, community-dwelling older adults showed that low mtDNAcn was associated with high inflammatory markers. ${ }^{35}$ Similarly, the reduction in mtDNAcn shown in the OCD group from the present study was significantly correlated with an increase in systemic inflammation as measured by the NLR. It has been suggested that cumulative damage to mitochondria and mtDNA induced by reactive oxygen species affects inflammatory responses and cellular senescence. ${ }^{36}$ In turn, inflammatory stress leads to enhanced reactive oxygen species production and cumulative damage to mtDNA, and the interplay between oxidative stress and mitochondrial dysfunction can result in energy failure, biological aging and the pathogenesis of age-related diseases. ${ }^{37,38}$ Thus, interplay between reduced mtDNAcn and elevated inflammation might contribute to the development of OCD.

On the other hand, mtDNAcn was not correlated with age in our sample, which consisted mostly of young adults. Although there has been some evidence showing an age-related decline in mtDNAcn, ${ }^{39-41}$ several reports (including our findings) have found no association between mtDNAcn and 
age. ${ }^{42-44}$ This inconsistency in findings may be because of different age groups used and a narrow age range among participants. Findings from previous literature of age-related decline in mtDNAcn was observed for participants older than 50 years. ${ }^{39,45}$ In addition, although mtDNAcn quantification has been used as a biomarker for mitochondrial function and has been implicated in age-related diseases, ${ }^{46}$ it is not a direct measure of the functionality of individual mitochondria and may reflect oxidative stress states rather than cellular aging itself. ${ }^{44,47}$ Indeed, mtDNAcn as measured by qPCR, which reflects the relative quantity of mitochondrial DNA compared to the nuclear DNA in a sample, should be considered a proxy for the content of mitochondrial copies. Mixed findings have been also reported with respect to the direction of altered mtDNAcn in several neuropsychiatric disorders. One study showed a significant reduction in mtDNAcn in combatrelated posttraumatic stress disorder and an "inverted-U" relationship between mtDNAcn and symptom severity of posttraumatic stress disorder. ${ }^{17}$ In contrast, increased mtDNAcn has been reported in patients with attention-deficit/ hyperactivity disorder ${ }^{48}$ and major depression, ${ }^{49}$ and was associated with childhood trauma and lifetime psychopathology, ${ }^{50}$ suggesting that an increase in mtDNAcn might be a compensatory cellular response to mitochondrial dysfunction. ${ }^{51}$ Interestingly, a meta-analysis of mtDNAcn studies in bipolar disorder revealed no association between bipolar disorder and alterations in mtDNAcn, but Asian populations with bipolar disorder showed significantly reduced mtDNAcn compared to controls. ${ }^{18}$ This observed discrepancy in results might be related to differences among studies in methods for extracting DNA and measuring mtDNAcn. Further research using a reliable measure of mitochondrial function and oxidative stress is needed to determine the involvement of mitochondrial dysfunction and cellular aging in neuropsychiatric diseases such as OCD.

After controlling for age, body mass index and childhood trauma, female participants with OCD exhibited significantly shorter telomere length compared to healthy controls. Although there is little direct evidence of altered telomere length in OCD, some indirect evidence supports an association between telomere shortening and OCD. Increased oxidative stress and oxidative imbalance have been implicated in the pathophysiology of OCD, $, 2,52,53$ and it is well established that oxidative stress is associated with shortened telomere length. ${ }^{14}$ Furthermore, increased oxidative DNA damage has been noted in OCD, ${ }^{54}$ which is known to induce telomere attrition. ${ }^{55}$ In this study, we observed telomere shortening in female, but not male, patients with OCD, possibly because women are more vulnerable to OCD-associated telomere erosion or because of potential differences in clinical features between men and women. Because the findings from sex-stratified analyses cannot clarify the contribution of sex to the cellular aging process, future research is needed to help determine the role of sex in telomere biology and mitochondrial function, and their different associations with psychiatric disorders.

Although the mechanisms linking cellular aging markers to OCD remain to be elucidated, it is unlikely that mitochondrial dysfunction and telomere erosion affect OCD patho- genesis via independent pathways. There is emerging evidence that these are linked biologically and have a complicated telomere-mitochondrial relationship during the human aging process.$^{56}$ Consistent with the findings of this study, leukocyte telomere shortening and mtDNAcn have been positively intercorrelated in healthy individuals $^{57,58}$ and in pregnant women, ${ }^{59}$ suggesting coregulation of telomere biology and mitochondrial function. ${ }^{58}$ Telomere dysfunction is known to reciprocally affect altered mitochondrial biogenesis. ${ }^{60}$ In addition, both oxidative stress and telomere erosion may be induced by mitochondrial dysfunction in response to stress. ${ }^{61}$ Although the underlying mechanisms are not well understood, elevated stress and proinflammatory cytokines may induce both mitochondrial dysfunction and altered telomere biology. ${ }^{15}$ Further research elucidating the complicated telomere-mitochondrial relationship and its underlying mechanisms is required.

\section{Limitations}

In the present study, we measured mtDNAcn and telomere length in peripheral blood, and not in brain tissue. It is unclear whether peripheral biomarkers reflect brain status in neuropsychiatric disorders. Notably, a postmortem study found that people who had died by suicide had a higher mtDNAcn in peripheral blood but a lower mtDNAcn in the prefrontal cortex compared to controls. ${ }^{62}$ Shorter telomere length was also identified in both the peripheral blood and prefrontal cortex of the same people, showing an opposite relationship between telomere length and mtDNAcn in postmortem blood and brain tissue. However, whether these peripheral biomarkers accurately reflect neuronal biomarkers remains unclear, and this is a methodological limitation of human research involving clinical populations.

The present study had other limitations. First, the crosssectional design did not allow us to infer a causal relationship between OCD disease status and cellular aging markers. Second, we controlled for the possible effects of age, childhood trauma and body mass index, but did not consider other potential confounders that may affect oxidative stress, such as recent stressors, smoking, exercise and nutrition. Third, we did not assess other potentially related biomarkers such as oxidative stress markers and telomerase activity. Fourth, we measured only cellular mtDNAcn in leukocytes and did not detect circulating mtDNA levels in cell-free plasma, which may be released into the circulation by the mitochondria under stress. ${ }^{63}$ In addition, although we found no significant relationship between platelet counts and mtDNAcn among participants with OCD (data not shown), mtDNAcn measured using the extracted genomic DNA from whole blood can be affected by mtDNA in platelets. ${ }^{64}$ Finally, although the results of the present study did not show a significant effect of medication status on mtDNAcn or telomere length, psychotropic drug treatments may affect cellular aging. The influence of psychotropic medications on mitochondrial processes has been investigated mainly using animal studies and thus remains unclear. ${ }^{65}$ Future longitudinal studies including larger samples of drug-naive patients with 
OCD are needed to confirm the present findings and elucidate the role of mitochondrial dysfunction in the pathophysiology of OCD.

\section{Conclusion}

The present study is the first to demonstrate alterations in mtDNAcn in OCD. We also identified telomere shortening in women with OCD. Our results suggest that aging-associated molecular mechanisms may be important in the pathophysiology of OCD. Future studies involving larger cohorts are warranted to investigate the role of mitochondrial dysfunction and telomere length in OCD and elucidate the molecular mechanisms that underlie OCD-related pathophysiology.

Affiliations: From the Department of Psychiatry and Institute of Behavioural Science in Medicine, Yonsei University College of Medicine, Seoul, South Korea (Kang, S.-T. Kim, S.-J. Kim); the Department of Psychiatry, CHA Bundang Medical Centre, CHA University, Seongnam, Republic of Korea (Park); the Department of Biochemistry and Biophysics, UCSF School of Medicine, San Francisco, CA, USA (Lin); and the Department of Medical Education, Yonsei University College of Medicine, Seoul, South Korea (H.-W. Kim).

Funding: This work was supported by a National Research Foundation of Korea (NRF) grant funded by the Korean government (NRF2018R1A2B2007714 and NRF-2019R1A2C1084611). The funding source did not influence the study design, data collection, analysis and interpretation of data, writing of the report or the decision to submit the article for publication.

Competing interests: None declared.

Contributors: J.-I. Kang and S.-J. Kim designed the study. C.-I. Park and S.-J. Kim acquired the data, which J. Lin, S.-T. Kim, H.-W. Kim and S.-J. Kim analyzed. J.-I. Kang wrote the article, which C.-I. Park, J. Lin, S.-T. Kim, H.-W. Kim and S.-J. Kim reviewed. All authors approved the final version to be published and can certify that no other individuals not listed as authors have made substantial contributions to the paper.

Content licence: This is an Open Access article distributed in accordance with the terms of the Creative Commons Attribution (CC BY-NC-ND 4.0) licence, which permits use, distribution and reproduction in any medium, provided that the original publication is properly cited, the use is noncommercial (i.e., research or educational use), and no modifications or adaptations are made. See: https:/ / creativecommons.org/licenses/by-nc-nd/4.0/

\section{References}

1. Hirschtritt ME, Bloch MH, Mathews CA. obsessive-compulsive disorder: advances in diagnosis and treatment. JAMA 2017;317:1358-67.

2. Adams TG, Kelmendi B, Brake CA, et al. The role of stress in the pathogenesis and maintenance of obsessive-compulsive disorder. Chronic Stress (Thousand Oaks) 2018;2:2470547018758043.

3. Kandemir $\mathrm{H}$, Abuhandan $\mathrm{M}, \mathrm{Aksoy} \mathrm{N}$, et al. Oxidative imbalance in child and adolescent patients with obsessive compulsive disorder. J Psychiatr Res 2013;47:1831-4.

4. Selek S, Herken H, Bulut M, et al. Oxidative imbalance in obsessive compulsive disorder patients: a total evaluation of oxidantantioxidant status. Prog Neuropsychopharmacol Biol Psychiatry 2008; 32:487-91.

5. Alici D, Bulbul F, Virit O, et al. Evaluation of oxidative metabolism and oxidative DNA damage in patients with obsessive-compulsive disorder. Psychiatry Clin Neurosci 2016;70:109-15.

6. De Hert M, Correll CU, Bobes J, et al. Physical illness in patients with severe mental disorders. I. Prevalence, impact of medications and disparities in health care. World Psychiatry 2011;10:52-77.
7. De Hert M, Dekker JM, Wood D, et al. Cardiovascular disease and diabetes in people with severe mental illness position statement from the European Psychiatric Association (EPA), supported by the European Association for the Study of Diabetes (EASD) and the European Society of Cardiology (ESC). Eur Psychiatry 2009;24:412-24.

8. Witthauer C, A TG, Meyer AH, et al. Physical diseases among persons with obsessive compulsive symptoms and disorder: a general population study. Soc Psychiatry Psychiatr Epidemiol 2014;49:2013-22.

9. Aguglia A, Signorelli MS, Albert U, et al. The impact of general medical conditions in obsessive-compulsive disorder. Psychiatry Investig 2018;15:246-53.

10. Wolkowitz OM. Accelerated biological aging in serious mental disorders. World Psychiatry 2018;17:144-5.

11. Epel ES, Blackburn EH, Lin J, et al. Accelerated telomere shortening in response to life stress. Proc Natl Acad Sci U S A 2004;101: 17312-5.

12. Blackburn EH, Epel ES, Lin J. Human telomere biology: a contributory and interactive factor in aging, disease risks, and protection. Science 2015;350:1193-8

13. Vakonaki E, Tsiminikaki K, Plaitis S, et al. Common mental disorders and association with telomere length. Biomed Rep 2018;8:111-6.

14. Lindqvist D, Epel ES, Mellon SH, et al. Psychiatric disorders and leukocyte telomere length: underlying mechanisms linking mental illness with cellular aging. Neurosci Biobehav Rev 2015;55:333-64.

15. Picard M, McEwen BS. Psychological stress and mitochondria: a conceptual framework. Psychosom Med 2018;80:126-40.

16. Youle RJ, van der Bliek AM. Mitochondrial fission, fusion, and stress. Science 2012;337:1062-5.

17. Bersani FS, Morley C, Lindqvist D, et al. Mitochondrial DNA copy number is reduced in male combat veterans with PTSD. Prog Neuropsychopharmacol Biol Psychiatry 2016;64:10-7.

18. Yamaki N, Otsuka I, Numata S, et al. Mitochondrial DNA copy number of peripheral blood in bipolar disorder: the present study and a meta-analysis. Psychiatry Res 2018;269:115-7.

19. First MB, Spitzer R. L GM, et al. Structured clinical interview for $D S M-I V$ axis I disorders, clinical version (SCID-CV). Washington (DC): American Psychiatric Press, Inc.; 1996.

20. Cawthon RM. Telomere measurement by quantitative PCR. Nucleic Acids Res 2002;30:e47.

21. Lin J, Epel E, Cheon J, et al. Analyses and comparisons of telomerase activity and telomere length in human $\mathrm{T}$ and $\mathrm{B}$ cells: insights for epidemiology of telomere maintenance. J Immunol Methods 2010;352:71-80.

22. He L, Chinnery PF, Durham SE, et al. Detection and quantification of mitochondrial DNA deletions in individual cells by real-time PCR. Nucleic Acids Res 2002;30:e68.

23. Goodman WK, Price LH, Rasmussen SA, et al. The Yale-Brown Obsessive Compulsive Scale. I. Development, use, and reliability. Arch Gen Psychiatry 1989;46:1006-11.

24. Montgomery SA, Åsberg M. A new depression scale designed to be sensitive to change. Br J Psychiatry 1979;134:382-9.

25. Wang $\mathrm{X}$, Cui D, Wang Z, et al. Cross-sectional comparison of the clinical characteristics of adults with early-onset and late-onset obsessive compulsive disorder. J Affect Disord 2012;136:498-504.

26. Bremner JD, Bolus R, Mayer EA. Psychometric properties of the Early Trauma Inventory-Self Report. J Nerv Ment Dis 2007;195: 211-8.

27. Imtiaz F, Shafique K, Mirza SS, et al. Neutrophil lymphocyte ratio as a measure of systemic inflammation in prevalent chronic diseases in Asian population. Int Arch Med 2012;5:2.

28. Barrett EL, Richardson DS. Sex differences in telomeres and lifespan. Aging Cell 2011;10:913-21.

29. Price LH, Kao HT, Burgers DE, et al. Telomeres and early-life stress: an overview. Biol Psychiatry 2013;73:15-23.

30. Pérez LM, Pareja-Galeano H, Sanchis-Gomar F, et al. 'Adipaging': ageing and obesity share biological hallmarks related to a dysfunctional adipose tissue. J Physiol 2016;594:3187-207.

31. Lacey CJ, Salzberg MR. Obsessive-compulsive disorder with mitochondrial disease. Psychosomatics 2008;49:540-2.

32. Orhan N, Kucukali CI, Cakir U, et al. Genetic variants in nuclearencoded mitochondrial proteins are associated with oxidative stress in obsessive compulsive disorders. J Psychiatr Res 2012; $46: 212-8$ 
33. Picard M, McEwen BS, Epel ES, et al. An energetic view of stress: focus on mitochondria. Front Neuroendocrinol 2018;49:72-85.

34. Mohanty A, Tiwari-Pandey R, Pandey NR. Mitochondria: the indispensable players in innate immunity and guardians of the inflammatory response. J Cell Commun Signal 2019;13:303-18.

35. Wu IC, Lin CC, Liu CS, et al. Interrelations between mitochondrial DNA copy number and inflammation in older adults. J Gerontol A Biol Sci Med Sci 2017;72:937-44.

36. Cui H, Kong Y, Zhang H. Oxidative stress, mitochondrial dysfunction, and aging. J Signal Transduct 2012;2012:646354.

37. Chakrabarti S, Munshi S, Banerjee K, et al. Mitochondrial dysfunction during brain aging: role of oxidative stress and modulation by antioxidant supplementation. Aging Dis 2011;2:242-56.

38. Mohanty A, Tiwari-Pandey R, Pandey NR. Mitochondria: the indispensable players in innate immunity and guardians of the inflammatory response. J Cell Commun Signal 2019;13:303-18.

39. Mengel-From J, Thinggaard $\mathrm{M}$, Dalgå̆rd $\mathrm{C}$, et al. Mitochondrial DNA copy number in peripheral blood cells declines with age and is associated with general health among elderly. Hum Genet 2014;133:1149-59.

40. Dolcini J, Wu H, Nwanaji-Enwerem JC, et al. Mitochondria and aging in older individuals: an analysis of DNA methylation age metrics, leukocyte telomere length, and mitochondrial DNA copy number in the VA normative aging study. Aging (Albany NY) 2020; 12:2070-83

41. Zhang $\mathrm{R}$, Wang $\mathrm{Y}, \mathrm{Ye} \mathrm{K}$, et al. Independent impacts of aging on mitochondrial DNA quantity and quality in humans. BMC Genomics 2017;18:890.

42. Vyas CM, Hazra A, Chang SC, et al. Pilot study of DNA methylation, molecular aging markers and measures of health and wellbeing in aging. Transl Psychiatry 2019;9:118.

43. Fries GR, Bauer IE, Scaini G, et al. Accelerated epigenetic aging and mitochondrial DNA copy number in bipolar disorder. Transl Psychiatry 2017;7:1283.

44. Vyas CM, Ogata S, Reynolds CF III, et al. Lifestyle and behavioral factors and mitochondrial DNA copy number in a diverse cohort of mid-life and older adults. PLoS One 2020;15:e237235.

45. Knez J, Winckelmans E, Plusquin M, et al. Correlates of peripheral blood mitochondrial dna content in a general population. Am J Epidemiol 2016;183:138-46.

46. Castellani CA, Longchamps RJ, Sun J, et al. Thinking outside the nucleus: mitochondrial DNA copy number in health and disease. Mitochondrion 2020;53:214-23

47. Movassaghi S, Jafari S, Falahati K, et al. Quantification of mitochondrial DNA damage and copy number in circulating blood of patients with systemic sclerosis by a qPCR-based assay. An Bras Dermatol 2020;95:314-9.

48. Kim JI, Lee S-Y, Park M, et al. Peripheral mitochondrial DNA copy number is increased in Korean attention-deficit hyperactivity disorder patients. Front Psychiatry 2019;10:506.
49. Chung JK, Lee SY, Park M, et al. Investigation of mitochondrial DNA copy number in patients with major depressive disorder. Psychiatry Res 2019;282:112616.

50. Tyrka AR, Parade SH, Price LH, et al. Alterations of mitochondrial DNA copy number and telomere length with early adversity and psychopathology. Biol Psychiatry 2016;79:78-86.

51. Picard M, Prather AA, Puterman E, et al. A mitochondrial health index sensitive to mood and caregiving stress. Biol Psychiatry 2018; 84:9-17.

52. Atmaca M, Tezcan E, Kuloglu M, et al. Plasma nitrate values in patients with obsessive-compulsive disorder. Psychiatry Clin Neurosci 2005;59:621-3

53. Ersan S, Bakir S, Erdal Ersan E, et al. Examination of free radical metabolism and antioxidant defence system elements in patients with obsessive-compulsive disorder. Prog Neuropsychopharmacol Biol Psychiatry 2006:30:1039-42.

54. Alici D, Bulbul F, Virit O, et al. Evaluation of oxidative metabolism and oxidative DNA damage in patients with obsessive-compulsive disorder. Psychiatry Clin Neurosci 2016;70:109-15.

55. Barnes RP, Fouquerel E, Opresko PL. The impact of oxidative DNA damage and stress on telomere homeostasis. Mech Ageing Dev 2019;177:37-45

56. Zole E, Ranka R. Mitochondria, its DNA and telomeres in ageing and human population. Biogerontology 2018;19:189-208.

57. Kim JH, Kim HK, Ko JH, et al. The relationship between leukocyte mitochondrial DNA copy number and telomere length in communitydwelling elderly women. PLoS One 2013;8:e67227.

58. Tyrka AR, Carpenter LL, Kao HT, et al. Association of telomere length and mitochondrial DNA copy number in a community sample of healthy adults. Exp Gerontol 2015;66:17-20.

59. Qiu C, Enquobahrie DA, Gelaye B, et al. The association between leukocyte telomere length and mitochondrial DNA copy number in pregnant women: a pilot study. Clin Lab 2015;61:363-9.

60. Sahin E, Colla S, Liesa M, et al. Telomere dysfunction induces metabolic and mitochondrial compromise. Nature 2011;470:359-65.

61. Picard M, Juster RP, McEwen BS. Mitochondrial allostatic load puts the 'gluc' back in glucocorticoids. Nat Rev Endocrinol 2014;10:303-10.

62. Otsuka I, Izumi T, Boku S, et al. Aberrant telomere length and mitochondrial DNA copy number in suicide completers. Sci Rep 2017;7:3176.

63. Lindqvist D, Wolkowitz OM, Picard M, et al. Circulating cell-free mitochondrial DNA, but not leukocyte mitochondrial DNA copy number, is elevated in major depressive disorder. Neuropsychopharmacology 2018;43:1557-64.

64. Hurtado-Roca Y, Ledesma M, Gonzalez-Lazaro M, et al. Adjusting MtDNA quantification in whole blood for peripheral blood platelet and leukocyte counts. PLoS One 2016;11:e163770.

65. Adzic M, Brkic Z, Bulajic S, et al. Antidepressant action on mitochondrial dysfunction in psychiatric disorders. Drug Dev Res 2016;77:400-6. 